19 Revue d'histoire du XIXe siècle

Société d'histoire de la révolution de 1848 et des

révolutions du XIXe siècle

$63 \mid 2021$

Puissances de la Commune

\title{
Introduction. Persistances de la Commune
}

\section{Quentin Deluermoz et Éric Fournier}

\section{OpenEdition \\ Journals}

Édition électronique

URL : https://journals.openedition.org/rh19/7772

DOI : $10.4000 /$ rh 19.7772

ISSN : $1777-5329$

Éditeur

La Société de 1848

Édition imprimée

Date de publication : 1 décembre 2021

Pagination : 9-19

ISSN : 1265-1354

Référence électronique

Quentin Deluermoz et Éric Fournier, «Introduction. Persistances de la Commune », Revue d'histoire du XIXe siècle [En ligne], 63 | 2021, mis en ligne le 01 janvier 2022, consulté le 21 février 2022. URL : http:// journals.openedition.org/rh19/7772 ; DOI : https://doi.org/10.4000/rh19.7772 


\section{QUENTIN DELUERMOZ, ÉRIC FOURNIER}

\section{Introduction \\ Persistances de la Commune}

Le $150^{\mathrm{e}}$ anniversaire de la Commune de Paris a surpris par l'intensité de ses manifestations, artistiques, festives, scientifiques et politiques, et ce d'autant plus qu'il a eu lieu à l'ombre des contraintes sanitaires de la pandémie - le troisième confinement de l'Île-de-France ayant été décrété, hasard de temporalités disjointes, le 18 mars 2021. Ce confinement a également contribué à assourdir la conflictualité politique de la mémoire de la Commune sans parvenir à l'étouffer, loin de là - autre fait notable. Car si la Commune est redevenue depuis quelques années une référence pour les luttes sociales les plus vives, traitées dans ce dossier par Ludivine Bantigny et Paula Cossart, force est de constater qu'à droite, elle ne se suscitait plus qu'une indifférence polie. Entrant en résonance avec le $150^{\mathrm{e}}$ anniversaire, le possible classement de la basilique du Sacré-Cœur de Montmartre aux monuments historiques a déplacé la querelle sur le terrain de l'anticléricalisme et ravivé les clivages gauche/droite, dont le point culminant aura été la montée au Mur des Fédérés, le 29 mai 2021, sans doute la plus importante manifestation unitaire au Père-Lachaise depuis le centenaire de $1971^{1}$. Ce même jour, une procession catholique en hommage aux otages de la Commune était autorisée, à Ménilmontant, par la préfecture de Police. Chahutée par le quartier, la procession s'est réfugiée dans l'église de Notre-Dame-des-Otages. Quelques heures plus tard, la police avait "nassé" pendant deux heures un débat animé par deux des auteurs de ce dossier dans une librairie attenante à l'église, dont le public avait eu le seul tort d'entonner la chanson La Semaine sanglante à la vue de la procession ${ }^{2}$.

Si une telle conflictualité peut rappeler ce qui se jouait en 1971, elle contraste avec le calme du $140^{\mathrm{e}}$ anniversaire en 2011. Celui-ci s'était distingué en effet par le dynamisme retrouvé d'une recherche universitaire, de plus en plus internationale, comme le soulignait le colloque de Narbonne (2426 mars 2011), à partir duquel Marc César et Laure Godineau ont dirigé le

1. Éric Fournier, La Commune n'est pas morte. Les usages politiques du passé de 1871 à nos jours, Montreuil, Libertalia, 2013.

2. "Est-il vrai que la police a "nassé" une librairie qui organisait un débat sur la Commune?", Libération, mis en ligne le 31 mai 2021. 
livre collectif, La Commune de 1871 : une relecture, paru en décembre 2019. Il signalait l'importance des chantiers menés sur les Communes depuis une quinzaine d'années, tout en inaugurant les parutions historiographiques liées au $150^{\mathrm{e}}$ anniversaire. Après le changement radical des perspectives historiographiques, par les travaux de Lefebvre ou de Rougerie, autour du $100^{\mathrm{e}}$ anniversaire et le déploiement de la recherche autour du 140, on aurait pu croire un peu hâtivement l'objet Commune cerné, délimité, stabilisé, sinon historiographiquement froid. L'actualité éditoriale de l'année 2021 a bousculé cette idée reçue. Si l'ouvrage coordonné par Michel Cordillot, $\mathrm{La}$ Commune de Paris 1871. Les acteurs, l'événement, les lieux se distingue comme une monumentale synthèse de l'état le plus récent de la recherche, son succès et sa visibilité sont en partie dus à l'importance des pages "débats et controverses", signalées par plusieurs recensions dans les médias généralistes, qui soulignent que l'histoire de la Commune est encore en mouvement ${ }^{3}$. Par ailleurs, ce dynamisme de la recherche peut prendre des formes plus classiques, telle la biographie de Léo Frankel par Julien Chuzeville ${ }^{4}$; ou la réédition remarquée par Chloé Leprince du livre d'Édith Tomas Les Pétroleuses (1963), que l'on aurait pu croire daté, mais à qui les enjeux sociaux du temps présent ont donné une nouvelle actualité. À l'inverse, il peut être en phase avec les nouvelles directions de la recherche, en s'interrogeant, par exemple, sur les expériences «au ras du sol» ou en revenant sur la question de la profondeur historique, voire anthropologique à l'œuvre dans l'événement. Même chose pour l'exploration des connexions et horizons spatiaux, connus ou inattendus, européens, impériaux ou globaux, que génère et dans lesquels s'inscrit l'insurrection parisienne - au point que l'on peut parler d'un «moment Commune» de 1870-18715. Des formes narratives nouvelles, à des fins de transmission ou d'organisation des connaissances, ont continué d'être explorées, roman, bande dessinée, chaînes YouTube ou animations ${ }^{6}$. Enfin, pour finir ce bref tour d'horizon du dynamisme de la recherche, il faut mentionner la diversité des manifestations scientifiques ${ }^{7}$. Plus singulièrement, à l'échelle mondiale, étant donné les contraintes sanitaires, le $150^{e}$ anniversaire

3. Marc César, Laure Godineau (dir.), La Commune de 1871 : une relecture, Grane, Créaphis, 2019; Michel Cordillot (dir.), La Commune de Paris 1871. Les acteurs, l'événement, les lieux, Paris, Éditions de l'Atelier, 2021.

4. Julien Chuzeville, Léo Frankel. Communard sans frontières, Montreuil, Libertalia, 2021.

5. Sur ces différentes dimensions: Quentin Deluermoz, Commune(s), 1870-1871, une traversée des mondes au XIXe siècle, Paris, Le Seuil, 2020.

6. Citons parmi de nombreux exemples, la bande dessinée puis le film d'animation de Raphaël Meyssan, Les Damnés de la Commune (Delcourt, 2017-2019, et Arte, 2021) ; le film d'animation La Boîte noire, de Joris Clerté et Laure Godineau (Doncvoilà Productions/Lardux Films/Origine Films, 2020); la série radio La Commune de Paris, dernière révolution avant la République, par Anaïs Kien et Somany $\mathrm{Na}$ (France culture, 2020), l'œuvre artistique A Bundle of Silences, 1871 (Ana Maria Gómez López, Milena Bonilla, Amsterdam) ou encore sur la chaîne Twich, l'émission Nota Bene consacrée à la Commune, avec Éric Fournier qui a comptabilisé près de 120000 vues.

7. Par exemple : la conférence du 10 octobre 2019, à Dartmouth College, Hanover NH, organisée par Robert St Clair et Seth Widden, "La Commune n'est pas morte!/Commune's Not Dead", Nineteenth-Century French Studies, vol. 49, 2021/3-4; Laura Forster, Laura O’Brien, Tom Stammers, 
de la Commune restera marqué par des possibles non advenus, des colloques qui auraient dû avoir lieu mais ont été annulés, notamment en Grèce, en Grande-Bretagne, aux États-Unis, au Mexique ou au Portugal.

Insistons : ce qui est notable avec la Commune est sa capacité à produire encore de nouvelles connaissances, tant l'événement semble balisé, et enserré depuis plus d'un siècle dans des grilles d'interprétations successives, certes parfois en concurrence, mais qui chacune ont leur capacité heuristique. La bibliographie réunie en 2006 par Robert Le Quillec proposait près de 600 pages et 5000 entrées : romans, mémoires, catalogues d'expositions, réflexions philosophiques, et bien sûr travaux de recherches, qu'ils soient français, américains, britanniques, russes, espagnols, allemands, chinois ${ }^{8}$. Une vie ne suffirait pas à les lire. La Commune se révèle un véritable monument scientifique, intellectuel et politique des $\mathrm{XIX}^{\mathrm{e}}$-XXI ${ }^{\mathrm{e}}$ siècles, tout en ne cessant d'aiguiser, à chaque moment, le problème historique qu'elle représente ${ }^{9}$. Un détour historiographique s'impose pour en prendre une plus juste mesure.

En France, la Commune est rapidement devenue un objet privilégié de l'histoire dite de gauche, républicaine ou socialiste ${ }^{10}$ - puis dans l'entre-deuxguerres, de l'histoire d'inspiration marxiste, ou inspirée des questionnements marxistes, en particulier dans le cadre de l'histoire économique et sociale. Le principal effort académique date des années 1950-1970. Le problème posé est de savoir comment placer la Commune de Paris dans l'histoire du mouvement ouvrier telle qu'elle est alors pensée : elle semble à la fois le crépuscule des révolutions du xIx ${ }^{\mathrm{e}}$ siècle, ouverte par la Révolution française, et, par son gouvernement à majorité ouvrière, l'aurore du mouvement ouvrier moderne, censé être plus structuré en termes d'organisation ou d'idéologie - et avoir été réalisé en 1917. Les travaux pistent alors les traces d'une éventuelle avantgarde ou s'intéressent au rôle des idéologies (qu'ils trouvent brouillonnes ${ }^{11}$ ). Une autre série étudie les échos de la Commune dans différents pays (comme l'Allemagne, la Grande-Bretagne, la Hongrie, l'Amérique latine) pour détecter son rôle dans le passage d'un âge "romantique» de la révolte à ces luttes dites précisément «modernes» du Xx ${ }^{e}$ siècle ${ }^{12}$. Ces travaux sont à l'évidence

"The Commune and its Others: an Outsider's History of 1871", Durham, Durham University, 3-4 juin 2021.

8. Robert Le Quillec, Bibliographie critique de la Commune de Paris, Paris, La Boutique de l'histoire, 2006.

9. Une présentation plus détaillée de cette historiographie, au seuil du cent-cinquantenaire, in Quentin Deluermoz, Commune(s), op. cit.

10. Jacques Rougerie, "La Commune et la gauche», in Jean-Jacques Becker, Gilles Candar (dir.), Histoire des gauches en France, t. 1 : L'Héritage du XIXe siècle, Paris, La Découverte, 2004, p. 95-112.

11. Pour ces décennies: Georges Bourgin, La Commune, QSJ, Paris, PUF, 1953; Charles Rihs, La Commune de Paris, ses structures et ses doctrines, Genève, Droz, 1955; Jean Bruhat, Jean Dautry, Émile Tersen, La Commune de Paris, Paris, Éditions sociales, 1960.

12. Liste tout sauf exhaustive : Eberhard Hackethal, «Der historische Platz der Pariser Kommune im praktiken Wirken und theoretischen Denken der zeitgenössichen deutschen Arbeiterbewegungen (1871-1878)", Jahrbuch für Geschichte (DDR), 1967/2, p. 75-122; Frank Strobl, "Die Pariser Kommune und die österreichische Arbeiterbewegung", Weg und Ziel, 1956/3; Samuel Berntsein, "The Impact of the Paris Commune in the United States", The Massachusetts Review, vol. 12, 
marqués par le contexte de la Guerre froide et pris dans une orientation intellectuelle et politique spécifique, mais ils sont loin de n'en n'être qu'un reflet. Et de fait, des connaissances sont établies et les interprétations s'affinent.

En 1971, comme le bicentenaire pour la Révolution française, le centenaire de la Commune marque un moment important dans l'inflexion des problématiques et dans la connaissance de l'événement.

Car en même temps, dans les années 1960, d'autres lectures de la Commune étaient avancées. Elles venaient davantage de la sociologie, de la philosophie ou de courants politiques radicaux - opposés au stalinisme, libertaires ou inspirés par les «années 68 ». En 1969 le sociologue André Découflé invitait à couper le lien entre la Commune et les révolutions antérieures pour promouvoir sa spontanéité et sa dimension profondément libertaire ${ }^{13}$. Le sociologue et philosophe Henri Lefebvre a lui aussi insisté sur cette dimension anarchisante de la Commune, en l'associant plus particulièrement au fait urbain. L'événement parisien correspondait bien à ce qu'il formulait alors comme le "droit à la ville», la revendication d'une qualité de vie et d'une créativité, opposées à l'urbanisme et à l'emprise commerciale ${ }^{14}$. Ses réflexions ont ensuite nourri, même indirectement, un autre courant de recherche, situé aux États-Unis. Il prend place dans les forts renouvellements de la sociologie historique des années 1970-1980. L'insurrection parisienne y est davantage analysée comme le révélateur des crises internes du capitalisme et de la modernité urbaine : la Commune serait peut-être un mouvement urbain autant voire plus que le fruit de la lutte des classes ${ }^{15}$. L'historien-sociologue Roger Gould a alors proposé dans Insurgent Identities ${ }^{16}$, à partir d'une analyse des réseaux, une issue à ce débat en suggérant une historicité des natures insurrectionnelles : la révolution de juin 1848 serait plus politique, les acteurs et actrices mobilisant davantage sur une identité de classe, et la Commune plus urbaine, les insurgés défendant plutôt une certaine notion de communauté urbaine. Ces conclusions ont toutefois été vite contestées par les spécialistes de la Commune ${ }^{17}$.

1971/3, p. 435-446. S'ajoutent les travaux importants, pour la Suisse, de Marc Vuilleumier et, pour la Belgique, de Jan Dondth et Daisy Devrée. L'enquête accompagne alors souvent celle sur la Première Internationale : La Première Internationale : l'institution, l'implantation, le rayonnement (Paris, 16-18 novembre 1964), Paris, Éditions du CNRS, 1968; Julian P. W. Archer, The First International in France, 1864-1872: its Origins, Theories and Impact, Lanham, University Press of America, 1997.

13. André Découflé, La Commune de Paris (1871) : révolution populaire et pouvoir révolutionnaire, Paris, Éditions Cujas, 1969; id., Sociologie des révolutions, Que sais-je?, Paris, Presses universitaires de France, 1968.

14. Henri Lefebvre, La Proclamation de la Commune, Paris, Gallimard, 1965; id., La Révolution urbaine, Paris, Gallimard, 1970; id., Critique de la vie quotidienne, t. 1, t. 2, Paris, L'Arche, 1961 ; id., Le Droit à la ville, Paris, Éditions Anthropos, 1968.

15. Manuel Castells, The City and the Grassroots, Berkeley, University of California Press, 1983; David Harvey, Consciousness and the Urban Experience, Baltimore, J. Hopkins University Press, 1985.

16. Roger Gould, Insurgent Identities: Class, Community and Protest in Paris from 1848 to the Commune, Chicago, University of California Press, 1995.

17. Critique dans Robert Tombs, "Les Communeux dans la ville : des analyses récentes à l'étranger», Le Mouvement Social, n 179, 1997/2, p. 93-105; Jacques Rougerie, "Autour de quelques 
Enfin, et les chevauchements sont constants, une dernière veine historiographique s'est développée en France à partir des années 1980 dans le cadre de l'histoire politique du fait républicain. Dès les débats sur l'amnistie, la Commune avait été appropriée par l'historiographie républicaine, à la fois gênée par ses «excès» et soucieuse de l'intégrer à son héritage. Un siècle plus tard, le problème consiste davantage à comprendre et à situer la Commune dans l'établissement de "la plus longue des républiques» (1870-1940) - caractérisée par l'État dans sa forme "moderne», la démocratie libérale et le régime républicain. François Furet fait «rentrer au port» la Révolution française en 1879, après la Commune et l'installation au pouvoir des républicains ${ }^{18}$, tandis que Maurice Agulhon, selon des intentions différentes, commence son histoire de la Troisième République au 4 septembre 1870, malgré l'épisode communard : il s'agit pour lui de souligner la longue histoire dont elle est alors le produit ${ }^{19}$. Reprise ensuite dans de nombreux travaux, cette perspective est, elle, portée par un schéma plus ou moins implicite de la modernisation : la Troisième République s'installerait parce que l'alphabétisation progresse, l'urbanisation se déploie, les transports et les relations à distance s'intensifient, etc. Les auteurs prennent certes soin de pointer des contradictions et de distinguer plusieurs types de modernisations (sociale, politique, économique, culturelle). Mais la Commune est isolée, sous la forme d'un hapax noyé dans une histoire plus structurée.

L'inflexion principale et encore sensible aujourd'hui est cependant venue de deux historiens, Robert Tombs et Jacques Rougerie, qui en plusieurs décennies ont renouvelé les questionnements. Leurs recherches ont opéré une sorte de "retour à l'histoire»: retour aux faits et aux perceptions des acteurs par l'exploitation de sources nouvelles; confrontation d'une connaissance fine des situations avec les généralisations idéologiques, théoriques ou sociologiques antérieures. Dès sa thèse, publiée sous le titre La Guerre contre Paris $^{20}$, l'historien britannique Robert Tombs portait l'attention sur le grand oublié de cette histoire, le camp versaillais. L'ensemble de ses travaux postérieurs vise par la suite, explicitement, à sortir la Commune de ses mythes pour la replacer à une plus incertaine «croisée des chemin $s^{21}$ ».

L'œuvre de Jacques Rougerie, proche dans ses manières de faire (proximité des archives, analyses à l'échelle des individus, attentions aux formes plurielles de mobilisation), est différente dans ses visées, et davantage tournée

livres étrangers ", in Claude Latta (dir.), La Commune de 1871, L'événement, les hommes et la mémoire, Saint-Étienne, Publications de l'Université de Saint-Étienne, 2004, p. 215-236. Une discussion de ces positions se trouve dans l'article d'Alexandre Frondizi du présent dossier.

18. François Furet, La Révolution, 1770-1880, Paris, Hachette, 1989.

19. Exemplaire à cet égard, Maurice Agulhon, La République, t. 1 : 1880-1932, Paris, Hachette, 1990 , p. 7-16.

20. Robert Tombs, La Guerre contre Paris, 1871, Paris, Aubier, 1997.

21. Robert Tombs, "Conclusions ", in Laure Godineau, Marc César (dir.), La Commune de Paris, op. cit, p. 355 . 
vers l'attention portée à la nature politique de l'expérience communaliste ${ }^{22}$. Dès les années 1960, grâce à une étude fine des journaux, rapports d'enquêtes ou dossiers des conseils de guerre, l'historien multipliait les mises au point contestant le rôle prépondérant de l'Association internationale des Travailleurs dans la Commune; il rappelait l'importance des notions de "peuple" et "d'antagonismes sociaux" pendant l'épisode ${ }^{23}$. Il en a surtout proposé au fil des ans une autre interprétation. Pour la comprendre, dit-il, il faut situer la Commune dans le riche républicanisme et socialisme du XIX siècle français, en particulier dans la continuité de l'expérience de la « république démocratique et sociale» exprimée dans les années 1848-1851 : selon cette dernière, le changement de forme politique (la république) ne saurait être dissocié de la transformation des rapports sociaux (travail et échange), grâce au relais notamment de l'association. Loin de se réduire à un brouillon d'idées, la Commune s'inscrit alors dans une autre histoire des mondes républicains et ouvriers des années 1840-1880, plus riche qu'on pense, et qui reste à explo$\operatorname{rer}^{24}$.

La révolte de Paris s'est ainsi trouvée ramenée à une certaine irréductibilité. Moins marquées par l'urgence théorique et politique, les recherches sur la Commune ont, à partir de la décennie 1990, davantage accompagné le renouvellement du questionnaire historien. Certaines connaissances sont complétées ou approfondies, comme celle des Communes de province ou des biographies de communards ${ }^{25}$. L'édition de sources et de mémoires commentées se poursuit ${ }^{26}$. Le contexte sociopolitique des années 1869-1870

22. Bien qu'aujourd'hui fort éloignés, du fait de positionnements politiques devenus inconciliables, les travaux de ces deux historiens, de la publication de la thèse de Tombs aux années 2010, entraient en résonance. Jacques Rougerie signale l'importance des travaux de Tombs dans la version augmentée de Paris libre 1871 en 2004 et reprend ses estimations basses des morts de la Semaine sanglante (avant de les discuter à nouveau), à partir de l'édition de 2014 de La Commune de 1871 (Presses universitaires de France, collection Que sais-je?). En 2012, ils écrivent, en outre, de concert, la très utile notice "La Commune de Paris», in Michel Pigenet, Danielle Tartakowsky (dir.), Histoire des mouvements sociaux en France (de 1814 à nos jours), Paris, La Découverte, 2012, p. 141-151.

23. Jacques Rougerie, «L'AIT et le mouvement ouvrier à Paris pendant les événements de 18701871 ", in Jacques Rougerie (dir.), 1871, Jalons pour une histoire de la Commune, Paris, Presses universitaires de France, 1973, p. 3-102; id., Paris Libre, 1871, Paris, Le Seuil, 2004 [1971].

24. Jacques Rougerie, La Commune de 1871, Paris, Presses universitaires de France, 2009 [1 ${ }^{\text {re }}$ éd. 1988]; id., «Entre le réel et l'utopie : République démocratique et sociale, Association, commune, Commune", in Laurent Colantonio, Caroline Fayolle (dir.), Genre et utopie. Avec Michèle RiotSarcey, Saint-Denis, Presses universitaires de Vincennes, 2014, p. 273-292.

25. Les premiers prolongent un mouvement antérieur : Jeanne Gaillard, Communes de province, Commune de Paris 1870-1871, Paris, Flammarion, 1971; Ronald Aminzade, Ballots and Barricads, Class Formation and Republican Politics in Nineteenth Century France, Princeton, Princeton University Press, 1993; Marc César, Mars 1871 : la Commune révolutionnaire de Narbonne, Sète, Éditions Singulières, 2008; Jacques Girault, Bordeaux et la Commune, 1870-1871, Périgueux, Éditeur Fanlac, 2009. Pour les biographies: Alain Dalotel, André Léo (1824-1900), la Junon de la Commune, Chauvigny, Association des publications chauvinoises, 2004; Michel Cordillot, Eugène Varlin, internationaliste et communard, Paris, Spartacus, 2016; Jacques Rougerie, Eugène Varlin. Aux origines du mouvement ouvrier, Paris, Éditions du Détour, 2019; Michèle Audin (dir.), Eugène Varlin, ouvrier relieur 1839-1871, Montreuil, Libertalia, 2019.

26. Par exemple : Jules Andrieu, Notes pour servir à l'histoire de la Commune de Paris, Paris, Libertalia, 2016. 
- et notamment le mouvement des "réunions publiques» - est également mieux connu, tout comme les espaces de sociabilités ${ }^{27}$, la garde nationale, les réseaux des quartiers révolutionnaires ou des échos de la Commune à l'étranger $^{28}$. D'autres regards ont été portés depuis une trentaine d'années : histoire des représentations et de l'art ${ }^{29}$ histoire des femmes et du genre ${ }^{30}$, de l'exil ${ }^{31}$, de la mémoire et des sensibilités ${ }^{32}$. Dans le premier domaine par exemple, Bertrand Tillier, dans La Révolution sans images, a proposé, au croisement de l'histoire de l'art, de l'histoire culturelle et de l'histoire politique, une étude de la créativité visuelle dans l'événement en même temps que de l'incapacité postérieure à fixer des images : une béance qui expliquerait sans doute la facilité avec laquelle l'événement a pu être réapproprié, non sans décalages, après $1880^{33}$. L'effort est ponctué de nouveaux colloques et synthèses ${ }^{34}$. La pertinence politique contemporaine de la Commune a même été récemment réévaluée : dans une interprétation soucieuse de faire jouer les récits-types de l'événement, la spécialiste de littérature Kristin Ross a lu dans la Commune les «germes» de positions politiques qui répondraient aux problèmes les plus actuels, tout en réévaluant l'importance de certaines notions («le luxe communal») et acteurs (Élisée Reclus). Tandis que Michèle Riot-Sarcey invitait dans un livre important à retrouver «l'historicité» qui lie la Commune aux

27. Alain Dalotel, Alain Faure, Jean-Claude Freiermuth, Aux origines de la Commune. Le mouvement des réunions publiques à Paris, 1868-1870, Paris, Maspero, 1980; Martin Philip Johnson, The Paradise of Association, Political Culture and Popular Organizations in the Paris Commune of 1871, Ann Arbor, The University of Michigan, 1996; Iain Chadwick, "Revolutionary Neighbourhoods and Networks during the Paris Commune of 1871", unpublished Phd Thesis, Oxford University, 2011.

28. Philipp Katz, From Appomattox to Montmartre: Americans and the Paris Commune, Cambridge, Mass., Harvard University Press, 1998; Michelle Coghlan, Sensational Internationalism: The Paris Commune and the Remapping of American Memory in the Long Nineteenth Century, Édimbourg, Edinburgh University Press, 2016.

29. Bertrand Tillier, La Commune de Paris, révolution sans images? Politique et représentations dans la France républicaine (1871-1914), Seyssel, Champ Vallon, 2004; Gonzalo J. Sanchez, Organizing Independence: The Artists Federation of the Paris Commune and its Legacy, 1871-1889, Lincoln, University of Nebraska Press 1997; Hollis Clayson, Paris in Despair. Art and Everyday Life under Siege (1870-71), Chicago/Londres, University of California Press, 2002.

30. Depuis l'ouvrage d'Édith Thomas, Les Pétroleuses, Paris, Gallimard, 1963, citons : Gay L. Gulliskson, Unruly Women of Paris: Images of the Paris Commune, Ithaca, NY, Cornell University Press, 1996; Carolyn J. Eichner, Surmounting the Barricades: Women in the Paris Commune. Bloomington, Indiana University Press, 2004.

31. Paul Martinez, "Communard Refugees in Great-Britain, 1871-1880", unpublished Phd Thesis, Sussex University, 1981; Laure Godineau, "Retour d'exil. Les anciens communards au début de la Troisième République», thèse d'histoire, sous la direction de Jean-Louis Robert, Université Paris 1 Panthéon-Sorbonne, 2000; Thomas C. Jones, Robert Tombs, "The French Left in Exile : quarantehuitards and communards in London, 1848-1880", in Debra Kelly, Martyn Cornick (eds.), A History of the French in London: Liberty, Equality, Opportunity, Londres, Institute of Historical Research, 2013, p. 165-191; Michel Cordillot, Utopistes et exilés du Nouveau Monde : des Français aux États-Unis, de 1848 à la Commune, Paris, Vendémiaire, 2013.

32. Éric Fournier, Paris en ruines. Du Paris Haussmannien au Paris communard, Paris, Imago, 2008.

33. Un prolongement de cette analyse, centré sur l'iconoclasme, in Emmanuel Fureix, L'Eil blessé. Politiques de l'iconoclasme après la Révolution française, Ceyzérieu, Champ Vallon, 2019.

34. Laure Godineau, La Commune de Paris par ceux qui l'ont vécu, Paris, Parigramme, 2010. Parmi les colloques importants, il faut signaler ceux de Perpignan (publié en 2000) et de Montbrison (publié en 2004) outre celui de Narbonne en 2011 déjà mentionné. 
révolutions précédentes, en particulier 1848, selon des discontinuités temporelles et politiques qu'il faut réapprendre à appréhender ${ }^{35}$. D'autres entreprises, davantage venues de l'anthropologie ou de la philosophie, comme "The Paris Commune as an Expansive Planetary Political Form", s'interrogent depuis 2020 sur les ressemblances entre différentes formes "communalistes» à une échelle globale et dans la longue durée, qui peuvent aussi expliquer les échos de l'événement parisien ${ }^{36}$.

La Commune, ainsi, se sera prêtée à toutes les grilles d'interprétations possibles, sans jamais y correspondre pleinement. Elle semble échapper aux grands récits comme aux tentatives de catalogages historiens. En cela, elle reste aujourd'hui un objet d'étude important pour la compréhension de l'histoire française, européenne et mondiale du XIX ${ }^{e}$ siècle comme pour la méthodologie et la vocation critique de l'histoire et des sciences sociales. La récente résurgence de la référence à la Commune dans les luttes sociales et politiques en France et à travers le monde, des Gilets jaunes aux mouvements Occupy en passant par les ZAD ne fait qu'accroître ce constat. Car il ne s'agit pas là seulement de continuité mémorielle mais bien à l'évidence de discontinuité des usages et d'une capacité de cette référence au passé à reprendre vie et sens dans des contextes historiques, géographiques et sociaux changeants ${ }^{37}$. La Commune offre ainsi une précieuse occasion de réfléchir aux notions «d'avancées historiographiques», de "problématiques émergentes» ou de sujets "classiques» : le couple archaïsme et modernité ne vaut sans doute pas plus pour les objets historiques que pour les périodes que l'on étudie, en particulier le XIx ${ }^{\mathrm{e}}$ siècle. Aussi et paradoxalement, malgré cet immense massif de travaux qui peut donner le vertige (mais aussi grâce aux connaissances qu'il a produites au cours des décennies) l'histoire de ces 72 jours parisiens, et avec elle des autres expériences communalistes de 1870-1871, recèle encore bien des thèmes et questions à explorer. Ce numéro spécial de la Revue d'histoire du XIXe siècle s'inscrit dans cette perspective. Il entend ainsi non pas dresser un énième bilan, mais dessiner des pistes de recherches possibles; et non pas affirmer un programme de recherche clé

35. Kristin Ross, L'Imaginaire de la Commune, Paris, La Fabrique, 2015 [1 $1^{\text {re }}$ éd. 2015]; Michèle Riot-Sarcey, Le Procès de la liberté. Une histoire souterraine du XIX siècle en France, Paris, La Découverte, 2016; voir aussi John Merriman, Massacre: The Life and Death of the Paris Commune, New York, Basic Books, 2014. Parmi les analyses issues de la philosophie qui permettent de repenser certains aspects des projets communards : Jean-François Dupeyron, À l'école de la Commune de Paris. L'histoire d'une autre école, Dijon, Éditions Raison et Passions, 2020.

36. Cf. [https://planetarycommune.org/] (consulté en octobre 2021), projet porté par l'Academy of Global Humanities and Critical Theory, University of Virginia, Duke University and the University of Bologna. Voir aussi les propositions du spécialiste de littérature comparée, Bruno Bosteels, sur "The Mexican Commune" depuis 2013.

37. Sur cette question, nous nous permettons de renvoyer aux propos recueillis par Guy Dreux et Christian Laval auprès de Quentin Deluermoz, "La Commune, espace-temps révolutionnaire. Entretien avec Quentin Deluermoz", Terrains/Théories, n 13, 2021 [https://journals.openedition. org/teth/3559?lang=en] (consulté en octobre 2021), et plus largement à l'ensemble du volume; ainsi que le numéro «La Commune est vivante!», Cahiers d'histoire. Revue d'histoire critique, n 148, 2021. 
en main, mais aiguiser les curiosités et montrer l'enrichissement continue des problématiques et des compréhensions. Il s'organise en deux parties, suivant en cela les travaux récents, qu'ils soient d'histoire, de sociologie ou de philosophie, qui ont invité à repenser le lien entre l'expérience communale parisienne de 1871 et l'idée communaliste (qui naît pour partie des appropriations dont la première fait l'objet).

La première concerne l'histoire de la Commune de 1871 elle-même. Dans son article, Alexandre Frondizi prolonge l'invitation faite par Jacques Rougerie, restée longtemps sans réponse, de mener une étude micro-historique de l'expérience révolutionnaire et de porter une attention fine aux dynamiques urbaines. Confortant au passage une chronologie importante du XIX ${ }^{\mathrm{e}}$ siècle français, 1830-1880, il montre comment l'attention portée aux espaces extérieurs puis intégrée au Paris haussmannien en 1860, comme les communes de Montmartre et de la Chapelle, permet de saisir de manière plus concrète les généalogies en jeu, de réfléchir à nouveaux frais à la question des «centres» de l'espace parisien en même temps qu'à celle, toujours non résolue, des nouages entre mobilisations urbaines, antagonismes sociaux et résurgences du passé dans le feu de 1871. Il remet ainsi en cause l'image classique de la reconquête de la ville post-haussmanienne et propose une conception enrichie de la transformation urbaine. Stephen Sawyer porte de son côté le regard vers le camp versaillais, resté étonnamment peu investi depuis le travail pionnier de Robert Tombs. Armé des outils de l'histoire intellectuelle et de l'histoire transnationale, il propose une nouvelle interprétation de la politique d'Adolphe Thiers en 1871. Il montre pour cela le rôle des circulations intellectuelles de part et d'autre de l'Atlantique (avec le modèle incarné par Abraham Lincoln) et invite surtout à s'interroger sur l'installation, en 1870-1880, dans une situation d'état d'exception, d'un État libéral fort. Si l'association peut sembler contradictoire à ce moment, cette forme politique est, en effet, vouée à durer.

La seconde partie s'intéresse, quant à elle, davantage aux résonances, usages, réinterprétations de la Commune et de l'idée communaliste. Jeanne Moisand, s'appuyant sur une enquête à paraître sur le mouvement cantonaliste espagnol de $1873^{38}$, propose à partir d'une histoire connectée, sociale et politique, nourrie des archives espagnoles, une réflexion fouillée sur «l'ombre de la Commune»: loin de la thématique ancienne de "l'influence» et de la diffusion française, l'étude montre les enjeux concrets de cette proximité temporelle comme des usages faits par les insurgé.e.s. L'historienne invite alors à s'interroger sur les hiérarchies mémorielles implicites qui ont par la suite porté l'éclairage sur l'une (Paris), et masquée l'autre, de l'autre côté des Pyrénées. Abordant le contemporain, l'article de la politiste

38. Jeanne Moisand, Se fédérer ou mourir. Carthagène, 1873, une Commune espagnole et ses mondes, Paris, Libertalia, 2021. 
Paula Cossart se confronte, lui, à une question émergente et délicate : quel est le lien, au-delà de la ressemblance des termes, entre les "Communes» de 1870-1871 et le "communalisme", inspiré des réflexions de Murray Bookchin, dans les années 1980 et plus largement les expériences de municipalisme libertaires des années 1960-202039 ? Elle propose pour y répondre un aperçu des résurgences de la Commune dans les luttes sociales du début $\mathrm{du} \mathrm{XXI}^{\mathrm{e}}$ siècle, et dresse une analyse serrée, au plus proche des textes, des concepts et des pratiques, sur les convergences et les écarts parfois inattendus entre l'événement historique du XIX ${ }^{e}$ siècle et ces réflexions et expérimentations postérieures. Enfin, Ludivine Bantigny propose une lecture plus sensible et engagée, directement ancrée dans les résurgences récentes, de «la Commune au présent». Dans la continuité des recherches en histoire et philosophie politique sur les discontinuités historiques et la force motrice des "passés vifs ", elle montre ce que mobiliser la référence à la Commune fait à notre présent, que ce soit l'intensification des réflexivités ou l'ouverture de temporalités historiques, et donc de possibles, autres. La Commune, souligne-t-elle, fait ainsi particulièrement sens aujourd'hui dans les combats de plus en plus nombreux invitant au dépassement du capitalisme et des gouvernances étatiques ou supra-étatiques ainsi, a contrario, qu'à l'élaboration de formes de vie plus justes.

Les autres rubriques de ce dossier complètent l'exploration des facettes de l'événement et de ses prolongements. Dans l'un de ses derniers textes, le regretté Jean-Claude Farcy, à qui la revue a rendu hommage dans son numéro 2020/2, présente les caractéristiques et les apports de la base de données sur la répression judiciaire de la Commune de Paris, base mise au point au terme d'un travail de longue haleine. Avec celle sur 1848, les historiennes et historiens bénéficient désormais d'un outil essentiel pour mener à bien de nouvelles enquêtes et, à n'en pas douter, renouveler encore dans l'avenir la connaissance de l'événement. La rubrique "le XIXe siècle vu d'ailleurs", présente, elle, d'autres réverbérations de l'événement communard, comme dans l'espace canadien étudié ici par Alban Bargain-Villéger, en Allemagne par Corentin Marion, ou encore au Pérou, sur lequel s'est penché Mario Tumen. Enfin, comme nous l'avons dit, les pistes qui restent à emprunter pour répondre à l'énigme posée par le «sphinx» qu'est la Commune sont nombreuses et excèdent celles pourtant riches proposées par les articles du dossier. Bien des connaissances et des efforts d'interprétations restent à mettre en ouvre. C'est pourquoi est ajouté à cette livraison un compte rendu du colloque "La Commune. L'histoire continue» qui s'est tenu en octobre 2021, coorganisé par Laure Godineau et les deux directeurs de ce dossier. Il a en effet pour particularité de présenter les résultats de

39. Par exemple : Murray Bookchin, Pour un municipalisme libertaire, Lyon, Atelier de création libertaire, 2018 [1 $1^{\text {re }}$ éd. 1984]. 
travaux en cours (master, doctorat ou postdoctorat), et donc de la recherche à venir. Sont abordés les mondes sociaux de la Commune, les connexions transnationales au cours de l'événement, la question des masculinités, le rôle des femmes comme celui des enfants, ou encore les enjeux de la représentation littéraire ou graphique de l'événement. Une manière de rappeler qu'à l'évidence, une fois passé le cent-cinquantenaire, la Commune n'est pas morte, en matière de recherche non plus.

Quentin Deluermoz est professeur d'histoire contemporaine à l'université de Paris et chercheur à l'ICT

(Identités, Cultures, Territoires)

Éric Fournier est maître de conférences à l'université Panthéon-Sorbonne et chercheur au CRH (Centre de recherche d'histoire du XIX' siècle) 